\title{
Os núcleos aplicativos e as línguas indígenas brasileiras
}

\author{
Applicative heads and the brazilian indigenous \\ languages
}

\author{
Marcia Maria Damaso Vieira \\ Museu Nacional/UFRJ
}

\begin{abstract}
In this work, we investigue the occurrence of applicative constructions in three Brazilian indigenous languages: Paumarí (Arawá family), Guarani and Tupinambá (Tupi-Guarani family), in the light of Pylkkänen's $(2000,2002)$ typology for applicative heads. We have observed the existence of two types of applicative morphemes in these languages: (i) those which select intransitive verbs, licensing syntactic objects with different theta roles (source, comitative, instrument, locative) - (High Applicatives); and (ii) those which are only attached to transitive verbs and which introduce objects with specific theta roles: source or goal/benefactive - (Low Applicatives). Through this typology, it was possible, then, to identify two types of applicative constructions in Tupi-Guarani: comitative causative and possessor stranding.
\end{abstract}

\section{Keywords}

Brazilian indigenous languages, Aplicative morphemes, Comitative, Possessor stranding 


\section{Resumo}

Neste trabalho, investigamos a ocorrência de construções aplicativas em três línguas indígenas brasileiras: Paumari (família Arawá), Guarani e Tupinambá (família Tupi-Guarani). À luz da tipologia de Pylkkänen $(2000,2002)$ para os núcleos aplicativos, identificamos dois tipos de estruturas aplicativas nas línguas observadas: (i) aquelas que só ocorrem com verbos intransitivos e que liberam objetos com papéis temáticos variados (fonte, comitativo, instrumento, etc.) (Aplicativas Altas); e (ii) as que só se manifestam com verbos transitivos e introduzem objetos com papéis temáticos específicos: fonte ou alvo / benefactivo (Aplicativas Baixas). Através dessa tipologia, foi possível identificar dois tipos de construções aplicativas em Tupi-Guarani, ainda não reconhecidos por outros investigadores dessas línguas, a saber: as causativas comitativas e as estruturas com ascensão do possuidor (Possessor Stranding).

\section{Palavras-chave}

Línguas indígenas brasileiras, Morfemas aplicativos, Comitativo, Ascensão do possuidor. 


\section{Introdução}

$\mathrm{E}$ xistem poucos trabalhos sobre as construções aplicativas verificadas em línguas indígenas brasileiras. Dentre estes, podemos citar Dourado (2002) que trata da derivação das aplicativas do Panará (família Jê), sob a ótica da Teoria Gerativa. A investigação das estruturas aplicativas é importante não só para a descrição e análise da morfologia e sintaxe dessas línguas, mas também para a questão de cunho teórico sobre o papel dos núcleos funcionais no licenciamento de argumentos. Sendo assim, se faz necessário investigar a ocorrência de tais construções em outras línguas brasileiras.

Neste trabalho, o nosso objetivo é, então, descrever e discutir as construções aplicativas de três línguas indígenas brasileiras: Paumari (família Arawá), Guarani e Tupinambá (família Tupi-Guarani), à luz da tipologia dos núcleos aplicativos proposta por Pylkkänen (2000, 2002), dentro do quadro da Morfologia Distribuída (MARANTZ, 1997).

Com base na parametrização dos núcleos aplicativos, sugerida por Pylkkänen, pudemos identificar diferentes tipos de estruturas aplicativas nas línguas aqui observadas. Em Vieira (2001), já havíamos sugerido que o afixo chamado de causativo-comitativo é, na verdade, um morfema aplicativo. Propomos aqui que as construções com ascensão do possuidor (Possessor Stranding $)^{1}$ do Tupinambá e do Guarani também devem ser analisadas como estruturas aplicativas com núcleo fonologicamente não realizado.

Apresentamos a seguir uma descrição das construções aplicativas verificadas nas línguas naturais, bem como um resumo da proposta de Pylkkänen para uma nova tipologia de estruturas aplicativas.

\section{As construções aplicativas: descrição e proposta de análise}

O termo aplicativo é empregado na literatura para descrever uma construção em que a adição de um afixo à morfologia verbal licencia um objeto 
extra. Em Chichewa (Bantu), por exemplo, o acréscimo do afixo aplicativo à morfologia verbal introduz um objeto. Nos exemplos abaixo, note-se que o verbo intransitivo “dançar”, em (1a), ao receber o sufixo -ir em (1b), se torna transitivo: ${ }^{2}$

Chichewa

1a. Atsikana a-na-vin-a

menina f-pass.-dançar-md

'A menina dançou'

b. Atsikana a-na-vin-ir-a mfumu

menina f.-pass.-dançar-APL.-md chefe

'A menina dançou (para) o chefe'

(BAKER, 1988, p. 258)

O sufixo aplicativo do Chichewa também pode ser adicionado a verbos transitivos, licenciando um segundo objeto. Observe em (2a) que o PP adjunto ("com uma lança”) no papel de instrumento se torna um objeto aplicativo em (2b), mediante o acréscimo de -ir ao verbo "cozinhar”:

Chichewa

2a. Kalúlú a-ku-phík-á maûngu ndí mkóndo a lebre m-pres.-cozinhar-md abóboras com lança 'A lebre está cozinhando abóboras com uma lança'

b. Kalúlú a-ku-phík-ír-a mkôndo maûngu lebre m-pres.-cozinhar-APL-md lança abóboras 'A lebre está cozinhando (com) uma lança as abóboras'

(MCHOMBO, 1998, p. 506)

Nem sempre o morfema aplicativo é visível na morfologia verbal. Em algumas línguas, como o Inglês, o afixo aplicativo não é fonologicamente expresso. Para explicar o fato de que o PP (to John) em (3a) ocorre como objeto em (3b), postula-se a existência de um morfema aplicativo abstrato, conforme ilustra a representação do verbo em (3c):

Inglês

3a. I baked a cake to John

b. I baked John a cake

c. [ bake[ ${ }_{\text {apl }} \varnothing\left[{ }_{\text {pass }}\right.$-ed]]] 
Existem diferentes tipos de estruturas aplicativas e de hipóteses que tentam explicá-las (BAKER 1988;1998; MCHOMBO, 1998 e JEONG, 2006), conforme veremos a seguir.

\subsection{Os tipos de construções aplicativas}

De acordo com Jeong (2006), na literatura, identificam-se dois tipos de estruturas aplicativas: as simétricas e as assimétricas. Baker (1988) discute a diferença entre esses dois tipos de construções. Nas aplicativas transitivas simétricas, tanto o objeto direto quanto o objeto aplicativo são sintaticamente ativos, podendo, por exemplo, engatilhar concordância verbal e tornar-se o sujeito da passiva. Nas aplicativas transitivas assimétricas, o objeto direto fica sintaticamente inerte, ao passo que o argumento aplicativo se comporta como o objeto sintático. Os dados em (4) e (5) ilustram a diferença entre esses dois tipos de aplicativas. Em Chaga (Bantu), língua com aplicativas simétricas, tanto o objeto aplicativo quanto o objeto direto podem se tornar sujeitos da passiva. Em Inglês, língua com aplicativas assimétricas, apenas o objeto aplicativo pode funcionar como sujeito da passiva, conforme indica a agramaticalidade de (5c):

$$
\begin{aligned}
& \text { Chaga } \\
& \text { 4a. n-a-i-lyì-à m-kà k-élyá } \\
& \text { 'Ele está comendo o alimento (para) a esposa' } \\
& \text { class-alimento class-pres-comer-APL-ps class-esposa } \\
& \text { c M-ka } \mathbf{a}_{\mathrm{i}} \text { n-a-i-lyi-i-o } \mathrm{t}_{\mathrm{i}} \quad \text { k-elya } \\
& \text { 'A esposa está tendo o alimento comido (para ela)' }
\end{aligned}
$$
5a. John baked Bill a cake
b. Bill ${ }_{i}$ was baked $t_{i}$ a cake.$$
\text { foc- f-pres-comer-APL-md class-esposa class-alimento }
$$$$
\text { b. K-ely } \text { i }_{i} \text { k-i-lyi-i-o m-ka ti }
$$$$
\text { 'O alimento está sendo comido (para) a esposa' }
$$$$
\text { class-esposa foc-suj-pres-comer-APL-ps class-alimento }
$$ 
Várias hipóteses foram postuladas na literatura para dar conta da derivação das estruturas aplicativas. Uma delas, defendida por Baker (1988), sugere que o morfema aplicativo corresponde a uma adposição que, ao se incorporar ao verbo, libera o seu complemento como um objeto independente. Neste trabalho, assumimos, porém, a hipótese de Pylkkänen (2000, 2002), por ser esta a que apresenta maior poder explicativo para os fatos relacionados às construções aplicativas das línguas aqui estudadas. Resumimos a seguir os pontos principais da proposta de Pylkkänen, embasada na Morfologia Distribuída.

\subsection{Aplicativas Altas e Baixas}

De acordo com a Morfologia Distribuída (MARANTZ,1997), teoria que assume que a formação das palavras ocorre na sintaxe, a estrutura argumental dos predicados tambémé definida na sintaxe. Os verbos, por exemplo, são constituídos por uma raiz lexical, um morfema categorizador ${ }^{3}$ e um núcleo funcional, como Voz (Voice), Causa ou Aplicativo (Apl). Tais elementos se combinam pelas operações sintáticas Concatenar (Merge) e Mover. As raízes lexicais podem selecionar ou não argumentos internos. Alguns núcleos funcionais são também responsáveis pela introdução de argumentos. Segundo Pylkkänen, o núcleo Voz, por exemplo, licencia o argumento externo que funciona como sujeito nas construções transitivas e inergativas. O núcleo Aplicativo introduz um objeto extra na construção em que se manifesta. Já o morfema Causa seleciona apenas um argumento com papel temático de evento. ${ }^{4}$

Com base em considerações léxico-semânticas, Pylkkänen (2002) postula a existência de dois tipos de núcleos aplicativos: o Aplicativo Alto e o Aplicativo Baixo. O primeiro relaciona um indivíduo ao evento descrito pelo VP. Em (4a), por exemplo, o sintagma "a esposa” se encontra em uma relação de benefactivo com o evento "comer”. O objeto aplicativo introduzido por esse núcleo pode também ter outras interpretações semânticas, a saber: instrumento, locativo, fonte, etc. Como denota uma relação entre um indivíduo e um evento, o núcleo Aplicativo Alto se combina sintaticamente com um VP como complemento e com um DP na posição de especificador, conforme ilustra a representação em (6):

6. Aplicativo alto: $\left[{ }_{\mathrm{vP}}\left[v\left[_{\mathrm{AplP}} \mathrm{DP}\left[_{\mathrm{Apl}}[\mathrm{VP} /(\mathrm{DP})]\right]\right]\right]\right]$ 
O núcleo Aplicativo Baixo denota uma relação de transferência de posse entre o objeto aplicativo e o objeto direto. Em (5a), “o bolo” é transferido para a posse de John, que assume o papel temático de alvo/benefactivo. Note-se que, dada a sua semântica, esse tipo de núcleo aplicativo nunca ocorre em estruturas sem um objeto direto. Essa relação de transferência de posse é expressa sintaticamente por dois DPs: um na posição de complemento do núcleo Apl e o outro na posição de especificador do sintagma AplP, como indica (7):

7. Aplicativo Baixo: $\left[{ }_{\mathrm{VP}} \mathrm{V}\left[_{\mathrm{APLP}} \mathrm{DP}\left[\left[_{\mathrm{Apl}} \mathrm{DP}\right]\right]\right]\right.$

Os termos Alto e Baixo estão, então, relacionados às posições onde os núcleos funcionais se concatenam. O Aplicativo Alto se agrega acima da raiz verbal, enquanto o Aplicativo Baixo é concatenado abaixo da raiz.

As construções Aplicativas Altas correspondem às aplicativas simétricas e as Baixas, às assimétricas. Pylkkänen (2000) menciona ainda uma outra diferença entre os objetos dos dois tipos de aplicativas: a possibilidade de estar ou não implícito. Nas Aplicativas Altas, o objeto direto pode estar implícito, como em (8). Já nas Aplicativas Baixas, o objeto direto deve estar sempre explícito devido à sua semântica de transferência de posse entre duas entidades. A ausência deste torna a sentença agramatical, como ilustra (9):

Chaga (Aplicativo Alto)

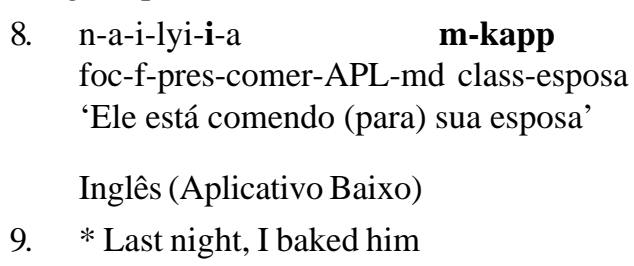

Existem ainda outros tipos de estruturas aplicativas não prototípicas. Trata-se das construções conhecidas na literatura como alçamento de possuidor e ascensão de possuidor, ${ }^{5}$ conforme sugerem Pylkkänen (2002) e Baker (1998).

\subsubsection{As construções com alçamento ou ascensão do possuidor}

As construções com alçamento do possuidor se caracterizam pela manifestação do possuidor e do elemento possuído como dois objetos sintáticos 
distintos. No exemplo(10a) da língua Kinyarwanda (Bantu), o possuidor genitivo se encontra dentro do DP objeto. Em (10b), o possuidor aparece em posição de objeto independente, sem que haja nenhuma modificação na morfologia verbal. Construções semelhantes são verificadas em Hebraico, como no exemplo (11):

\section{Kinyarwanda}

10a. Umugore y-a-vun-nye ukuboko k’ uumwaana mulher suj-pass-quebrar-asp braço de criança 'A mulher quebrou o braço da criança'

b. Umugore y-a-vun-nye umwaana ubukoko mulher suj-pass-quebrar-asp criança braço 'A mulher quebrou o braço da criança'

(BAKER, 1988, p. 270)

Hebraico

11. Ha-yalda kilkela le-Dan et há-radio a-menina estragou para- Dan acus. o-rádio 'A menina estragou o rádio (da posse de) Dan' (=The girl spoiled Dan's radio on him')'

(PYLKKÄNEN, 2002, p. 43)

Para Landau (1999) (cf. PYLKKÄNEN, 2002), na derivação dessas construções, o possuidor se desloca da posição de especificador do DP objeto para a posição de especificador do VP. ${ }^{6}$ Pylkkänen propõe, porém, que essas estruturas são, na verdade, Aplicativas Baixas que correspondem a orações com dois objetos. De acordo com a autora, em (11), o possuidor no caso dativo tem interpretação de fonte (from the possession of) e não de um mero possuidor. Trata-se, portanto, de um argumento licenciado por um núcleo aplicativo abstrato.

Uma evidência de que Dan em (11) não é o possuidor alçado da posição de especificador do DP objeto, vem da possibilidade de se acrescentar um outro possuidor à construção, conforme ilustra (12). Se a análise de Landau estivesse correta, não seria possível preencher tal posição com um outro sintagma possuidor: ${ }^{7}$

Hebraico

12. Gil šavar le-Rina et ha-miskafayim šel Sigal

Gil quebrou para-Rina acus os-óculos de Sigal

'Gil quebrou os óculos de Sigal em Rina'

(Gil broke Sigal’s glasses on Rina)

(PYLKKÄNEN, 2002, p. 46) 
Ainda segundo a investigadora, se o possuidor dativo estivesse interno ao DP em algum momento da derivação, ele poderia ser interpretado como agente em nominalizações, da mesma maneira que um possuidor genitivo. Observe que, em (13), o possuidor no caso genitivo (šel ha-cava) tem papel temático de agente. Já em (14), o possuidor dativo (la-cava) não pode ocorrer como o agente da nominalização, como indica a agramaticalidade da sentença:

Hebraico

13. Ha-haresa šel ha-cava et ha-ír a-destruição de o-exército acus a-cidade 'A destruição da cidade pelo exército' (The army's destruction of the city)

14. *Cilamti la-cava et ha-harisa šel ha-ir Eu-fotografei para-o exército acus a-destruição de a-cidade 'Eu fotografei a destruição da cidade pelo exército' (I photographed the army's destruction of the city')

(PYLKKÄNEN, 2002, p. 50)

Com base em evidências como as apresentadas acima, Pylkkänen conclui que as construções com possuidor dativo em Hebraico envolvem um núcleo Aplicativo Baixo, fonologicamente nulo, que introduz um argumento sintaticamente independente no papel de fonte.

Baker (1998) apresenta uma proposta de análise semelhante à de Pykkänen para as construções com as construções com ascensão do possuidor do Chukchee, como (15b) abaixo. Note-se que, neste caso, o elemento possuído é incorporado ao verbo, aparentemente deixando para trás o possuidor na posição de objeto:

Chukchee

15.a $\exists$ nan remkel'-in poyg-na maca-tku-nin ele(erg) convidado-poss lança-abs quebrar-iter-3sj/30bj 'Ele quebrou a lança do convidado'

b. ヨnan poyga-mca-tko-nen remkel'-na ele(erg) lança-quebrar-iter-3sj/3obj convidado-abs 'Ele quebrou a lança ( para(o malefício)) do convidado

(BAKER, 1998, p. 347)

Baker (1998, p. 348) tece o seguinte comentário sobre a sentença (15b): "it is perfectly reasonable to analyze 'guest' as an affected object added by some 
kind of applicative process, rather than as stranded possessor of the incorporated noun... 'guest' is not merely a possessor in [15b], but rather a malefactive applied object...” Sendo assim, uma melhor tradução para (15b) seria: “Ele quebrou a lança para (o malefício) do convidado”.

Dada essa visão geral sobre a tipologia dos morfemas aplicativos, passamos agora a descrever e discutir a manifestação das estruturas aplicativas em Paumari, Guarani e Tupinambá.

\section{As construções aplicativas nas Línguas Indígenas Brasileiras}

Os dados das três línguas aqui investigadas revelam a existência dos dois tipos de núcleos aplicativos sugeridos pela tipologia de Pylkkänen. Os Aplicativos Altos são verificados apenas com verbos intransitivos ou intransitivizados. Já os Aplicativos Baixos se agregam a verbos transitivos ou bitransitivos, como mostramos a seguir.

\subsection{Os morfemas Aplicativos Altos em Paumari}

Alíngua Paumari ${ }^{9}$ possui dois afixos para a expressão do núcleo Aplicativo Alto: va/vi- e-ka. O prefixo va/vi, chamado por Chapman (1978) de comitativo, ${ }^{10}$ se agrega a verbos intransitivos e licencia um objeto que pode assumir interpretações semânticas variadas: comitativo, fonte, locativo, instrumento, etc. Em (16a) e (16b), tem-se a ocorrência do verbo intransitivo “viajar”. Neste último exemplo, há um sintagma adjunto expresso pelo PP “com a minha criança”. Em (16c), com o acréscimo do afixo aplicativo à morfologia verbal, a construção torna-se transitiva, licenciando, assim, um objeto no papel de comitativo. ${ }^{11} \mathrm{O}$ mesmo procedimento pode ser verificado com o verbo intransitivo "chorar" em (17):

16a. a-adaha-hi

1sg.-viajar-md

'Eu viajei'

b. a-adaha-hi kodi-isai khama

1sg.-viajar-md minha-criança com

'Eu viajei com a minha criança' 
c. a-va-adaha-há ada isai 1sg-APL.- viajar-md dem. criança

'Eu viajei (com) a criança'

17a. o-asara-hi

1sg-chorar-md

'Eu chorei'

b. o-va-asara-ha ada isai

1sg-APLchorar-md dem criança

'Eu chorei (com)a criança'

(CHAPMAN, 1978, p. 36)

Em (18b), - va introduz um objeto aplicativo no papel de fonte:

18a. va-ihamahi-ki adani isai

3pl-zangado-md dem criança

'As crianças estão zangadas'

b. daroba bononi ra va-va-ihamahi-vini daroba fruta-acus 3pl-APL-zangado-tran

'Eles estavam zangados (a partir d)a fruta’' (CHAPMAN, 1978, p. 36)

O prefixo - $k a$, quando se agrega a verbos intransitivos, também licencia um objeto com papéis temático variados. Compare (17b) e (18b) com (19) e (20) abaixo:

19. o-ka-asara-há ada isai

1sg-APL-chorar-md dem criança

'Eu chorei (pel)o menino'

20. ho-ra ka-ihamahi-ha ada isai

1sg-acus APL-zangado-md dem criança

'O menino estava zangado (com)igo’ (CHAPMAN, 1978, p. 24)

Como vimos acima, o núcleo aplicativo do tipo Alto em Paumari só pode ocorrer com verbos intransitivos. Um verbo transitivo deve ser intransitivizado antes de receber tal morfema. Em (21a), o verbo transitivo "lavar" ocorre com o sintagma adjunto "com sabão", no papel de instrumento. Em (21b) esse verbo se intransitiviza pela adição do sufixo - $\boldsymbol{a}^{12}$ e mantém o adjunto. Já em (21c), o afixo aplicativo é acrescentado ao verbo intransitivizado e a construção torna-se transitiva novamente, tendo como objeto sintático o sintagma "sabão” no papel de instrumento: 
21a. Mamai-a bi-soko-ki hida makari sabão-a mamãe-erg. 3-lavar-md dem. roupa sabão-obl 'Mamãe lavou a roupa com sabão'

b. soko-a-ki hida mamai sabão-a lavar-intr.-md dem. mamãe sabão-com 'Mamãe lavou com sabão'

c. Mamai-a bi-vi-soko-a -vini ida sabão mamãe-erg. 3-APL.-lavar-int.-tran. dem. sabão 'Mamãe lavou (com) sabão'

(CHAPMAN, 1978, p. 38)

\subsection{O causativo-comitativo das línguas Tupi-Guarani}

Nas línguas da família Tupi-Guarani, o morfema -ro é tratado pelos investigadores como causativo comitativo. Vieira (2001) argumenta, contudo, que uma das funções desse morfema é a de licenciar um objeto. Esta propriedade o caracteriza como um afixo aplicativo. Note-se nos exemplos abaixo, que os sintagmas adjuntos que coocorrem com os verbos inergativos em (22b) e (23b) se tornam objetos em (22c) e (23c), mediante o acréscimo do afixo -ro à morfologia verbal: ${ }^{13}$

Guarani

22a. Ava o-ke homem 3-dormir 'O homem dormiu'

b. Ava o-ke mitã reve homem 3-dormir criança com 'O homem dormiu com a criança'

c. Ava o(gwe)-ro-ke mitã homem 3-APL.-dormir criança 'O homem dormiu (com) a criança'

23a. Xee a-po

Eu 1sg-pular

'Eu pulei'

b. Xee a-po mitã reve

Eu 1sg-pular criança com

'Eu pulei com a criança' 
c. Xee a-ro-po mitã

Eu 1sg-APL-pular criança

'Eu pulei (com) a criança'

Os dados do Tupinambá em (24) e (25) também ilustram a ocorrência do prefixo aplicativo (causativo comitativo) com verbos intransitivos, licenciando, então, um objeto:

Tupinambá

24. a-ro-pytá ygara

1sg-APL-ficar canoa

'Eu parei (com) a canoa'

25. a-ro-ker aoba

1sg-APLdormir roupa

'Eu durmo (com) a roupa'

(LEMOS BARBOSA, p. 196)

Para que -ro co-ocorra com um verbo transitivo nestas línguas, é preciso intransitivizá-lo primeiro, através de reflexivização ou de incorporação nominal, como mostram os dados do Tupinambá em (26). O verbo transitivo “ver” em (26a) é intransitivizado em (26b), através da incorporação do objeto. Pela adição do afixo aplicativo, "ver” é novamente transitivizado: ${ }^{14}$

Tupinambá

26a. ere-î-epîak ybák

2sg-3-ver céu

'Você viu o céu?'

b. ere-ro-ybák-epîak-pe mitanga?

2sg.-APL.-céu-ver-inter. criança

Você viu o céu (com) a criança?

(LEMOS BARBOSA, p. 199)

A transitivização do verbo pela adição do prefixo aplicativo é observada no exemplo do Guarani a seguir. Em (27), vê-se que o verbo com morfologia aplicativa pode ser causativizado com o sufixo-uka, exclusivo dos verbos transitivos nas línguas Tupi-Guarani:

Guarani

27. a-ro-ke-uka kunhã pe mitã

1sg.-APL.-dormir-caus. mulher para criança

'Eu fiz a mulher dormir(com) a criança' 
Também podemos observar que o aplicativo -ro introduz não só objetos comitativos, mas também objetos com papel temático de fonte, como exemplificam (28) e (29). Note-se que, em (29), o objeto aplicativo se encontra incorporado ao verbo: ${ }^{15}$

28. a-no-tin xe-sy

1sg-APL-vergonha 1sgposs-mãe

'Eu (me) envergonho (d)a minha mãe'

29. oro-Tupã-(e)ro-bîar

1pl-Tupã-APL-confiar

'Nós confiamos (em) Deus'

(LEMOS BARBOSA, p. 197)

O objeto aplicativo tem comportamento de objeto sintático já que pode licenciar um afixo de pessoa na morfologia verbal. Em (30), a forma portmanteau que indica que o objeto sintático é de $2^{\mathrm{a}}$ pessoa e o sujeito de $1^{\mathrm{a}}$ pessoa do singular é empregada:

Tupinambá

30. oro-(e)ro-por-ausub

1sg/2sg-APL-gente-amar

'Eu te fiz amar gente'

(LEMOS BARBOSA, p. 199)

Como pode ser observado nos exemplos acima, o simples fato de esses afixos - va/vi, -ka e -ro licenciarem um objeto e de se agregarem a verbos intransitivos, inclusive os inergativos, coloca-os na categoria dos núcleos Aplicativos Altos. Além disso, como a semântica desses morfemas relaciona um indivíduo ao evento expresso pelo VP, o objeto aplicativo pode ter diferentes interpretações, como: fonte, comitativo, locativo e instrumento, conforme prevê a tipologia de Pylkkänen.

Passamos agora para a apresentação das estruturas aplicativas, envolvendo o núcleo do tipo Baixo.

\subsection{Os núcleos Aplicativos Baixos}

O núcleo Aplicativo Baixo denota uma relação de transferência de posse direcional entre o objeto aplicativo e o objeto direto - da posse de (fonte) ou para a posse de (alvo/benefactivo), conforme sugere Pylkkänen (2000). As línguas 
aqui observadas também possuem construções aplicativas que refletem a presença de núcleos Aplicativos Baixos.

\subsubsection{O morfema ka-...-hi em Paumarí}

Em Paumarí, o morfema Aplicativo Baixo é formado pelo prefixo $\boldsymbol{k a}$ - e pelo sufixo - hi. Este morfema descontínuo se agrega a verbos transitivos, tornando-os bitransitivos. O objeto aplicativo licenciado tem sempre o papel de benefactivo ou alvo. Observe os dados em (31). (31a) exibe uma estrutura transitiva derivada pelo acréscimo do morfema causativo na-à raiz “morrer”. Em (31b), tem-se a manifestação de um benefactivo, "para mim", na condição de adjunto. Já em (31c), após a inserção do afixo aplicativo ao verbo, o sintagma no papel de alvo/ benefactivo se realiza como objeto no caso acusativo:

31a. Koko-a bi-na-abini-hi ida arakava tio-erg. 3-caus.-morrer md dem. galinha 'O titio matou a galinha'

b. Koko-a bi-na-abini-hi ida arakava kodi-moni tio-erg. 3-caus.-morrer-md dem. galinha mim para 'O titio matou a galinha para mim'

c. Ho-ra ka-abini-hi-vini hi-ki ida arakava me-acus. APL.-morrer-APL.-tran. aux. dem. galinha 'O titio matou uma galinha ( para) mim'

(CHAPMAN; DERBYSHIRE, 1991, p. 302)

Os dados a seguir ilustram outras ocorrências do núcleo Aplicativo Baixo em Paumari. Em todos os casos, a presença do afixo descontínuo indica direcionalidade de transferência entre o objeto direto e o objeto aplicativo:

32. Ho-ra ka-khora-hi-vini hini ida nami me-acus APL-cavar-APL-tran aux dem solo 'Ele cavou o solo (para) mim'

33. Gisi ho-ra ka-jaha-hi-hi ida gora bodoni Gisi 1sg-acus APL-limpar-APL-md dem casa dentro 'Gisi limpou o interior da casa (para) mim' (CHAPMAN; DERBYSHIRE, 1991, p. 302) 
Nessas construções, o objeto aplicativo é sintaticamente ativo. Já o objeto direto torna-se inerte para todos os processos. Trata-se, então, de uma estrutura aplicativa assimétrica. Segundo Chapman e Derbyshire (p. 302) “The semantic patient, which is the direct object in the normal transitive clause, becomes a DEMOTED OBJECT in the benefactive ditransitive construction..$^{16}$ This may be marked as such by the $-a$ DEMOTED OBJECT enclitic or it may occur postverbally with a demonstrative but no other marking." 17

Com base no acima exposto, podemos concluir que as estruturas contendo o morfema ka-...-hi envolvem o afixo Aplicativo Baixo (assimétrico).

\subsubsection{As construções com ascensão de possuidor em Tupi- Guarani}

Nas línguas da família Tupi-Guarani não é observada nenhuma construção envolvendo núcleos Aplicativos Baixos realizados fonologicamente. No entanto, seguindo as propostas de Baker (1998) e de Pylkkänen (2002) para as construções com alçamento ou ascensão de possuidor em outras línguas, sugerimos aqui que as sentenças do Tupinambá e do Guarani do mesmo tipo também envolvem um núcleo Aplicativo Baixo.

Compare os exemplos (34) e (35) com os de Chukchee repetidos a seguir como (36). Em (34b), (35b) e (36b),o elemento possuído é incorporado ao verbo, deixando o possuidor em posição de objeto. Pela interpretação de (34b), obtida a partir de sua tradução, é possível aventar a hipótese de que o DP na posição de objeto é um argumento no papel temático de alvo/ benefactivo e não um mero possuidor. Esta mesma análise é estendida para (35b). Já a interpretação do objeto aplicativo em (36b) é de malefactivo como proposto por Baker (1998): ${ }^{18}$

Tupinambá

34a. a-î-monhanag xe r-uba kó

1sg.-3-fazer 1sg poss rel.-pai roça para

'Eu faço a roça do meu pai'

(LEMOS BARBOSA, p. 207)

b. a-î-kó-monhang xe r-uba

1sg.-3-roça-fazer 1sg poss rel.-pai

'Eu faço a roça a (de) meu pai’

(LEMOS BARBOSA, p. 207) 
Guarani

35a. Xee a-joi ava'i py

Eu 1sg-lavar menino pé

'Eu lavei o pé do menino'

b. Xee a-py-joi ava'i

Eu 1sg-pé-lavar menino

‘Eu lavei o pé do menino’ (= ‘Eu lavei o pé em benefício do menino’)

Chukchee

36.a. ᄏnan remkel'-in pyg-na maca-tku-nin

ele(erg) convidado-poss lança-abs quebrar-iter-3sj/30bj

'Ele quebrou a lança do convidado'

b. ヨnan poyga-mca-tko-nen remkel'-na

ele(erg) lança-quebrar-iter-3sj/3obj convidado-abs

'Ele quebrou a lança ( para(o malefício)) do convidado

(BAKER, 1998, p. 347)

Observe ainda os exemplos do Tupinambá envolvendo o verbo “dar”. Em (37a) e (37b), o benefactivo é expresso como um PP. Quando o objeto direto é incorporado ao verbo, como em (37c), o benefactivo se mantém na forma de PP, uma vez que a construção se torna intransitiva:

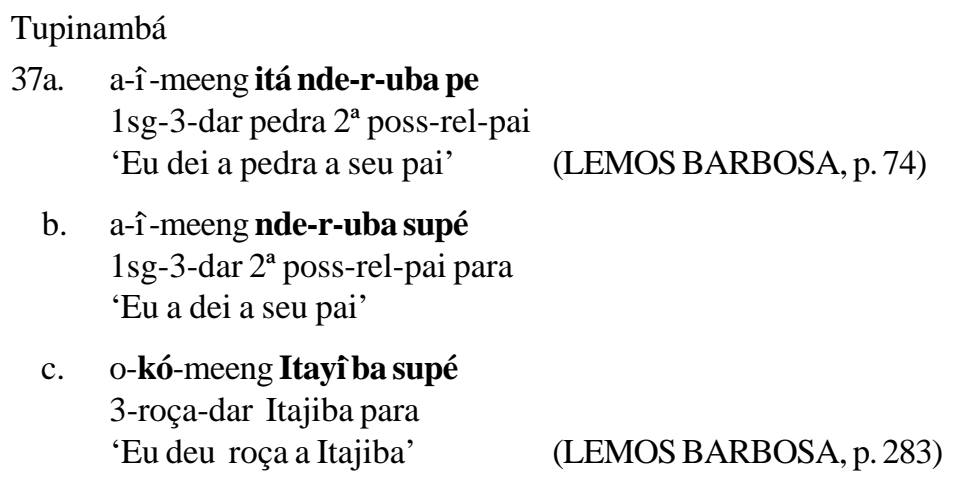

Existe, porém, a possibilidade de a estrutura com incorporação do objeto, como em (37c), tornar-se transitiva. Isto é observado pela presença do prefixo objetivo -îna morfologia verbal e pela supressão da posposição, como nos mostra (38). O objeto aí mantém a interpretação de alvo/benefactivo: 
Tupinambá

38. -î-kó-meeng Itayîba

3-3-roça-dar Itayî ba

'Ele deu roça a Itajiba'

(LEMOS BARBOSA, p. 283)

Estrutura semelhante a (38) é verificada em (34b), (35b) e em (39) a seguir. Esta última contém o verbo leve rung que só ocorre com o objeto direto incorporado. Neste caso, o objeto sintático é interpretado como alvo/benefactivo e não como possuidor:

Tupinambá

39. e-î-kó-rung nde mena

2imp-3-roça-fazer $2^{a}$ poss - marido

'Fazei a roça (para) o seu marido'

(LEMOS BARBOSA, p. 210)

Acreditamos, então, que as construções com ascensão de possuidor das línguas Tupi-Guarani envolvem um morfema aplicativo abstrato que licencia um objeto extra. Nos exemplos acima, o objeto licenciado tem interpretação de alvo/ benefactivo. Como tais aplicativas denotam uma relação de transferência de posse direcional (de (fonte) ou para (alvo/benefactivo)), sugerimos que o núcleo aí envolvido seja do tipo Baixo. Assim, em (40), “cobra” é um objeto aplicativo com papel de fonte (cortou a cabeça da (a partir da) cobra”). ${ }^{19} \mathrm{O}$ mesmo pode ser dito sobre (41) (furei a orelha da (a partir da) minha criação’):

Tupinambá

40. a-î-akang-ok mboî a
1sg-3-cabeça-cortar cobra
'Eu corto cabeça à cobra'

41a. a-î-kutuk xe-r-e-imbaba nami

1sg-3-furar 1sgposs-rel-animal orelha

'Eu furo a orelha da minha criação'

b. a-î-nami-kutuk xe-r-e-imbaba

1sg-3-orelha-furar 1sgposs-rel-animal

'Eu furo a orelha à minha criação' (LEMOS BARBOSA, p. 205-206)

Com base nas evidências fornecidas por Pylkkänen para o estatuto de objeto aplicativo do possuidor dativo em Hebraico, analisamos as estruturas com ascensão do possuidor das línguas Tupi-Guarani. Nas nominalizações, o DP 
possuidor sempre tem a interpretação de alvo/benefactivo, como ilustram os exemplos (42) e (43). (43) corresponde a um particípio passivo sem agente e o sufixo nominalizador - pyra/byra só pode fazer referência ao alvo/benefactivo que é passivizado porque é o único sintagma com estatuto de objeto sintático:

Tupinambá

42. Itayiba kó-meeng-aba

Itajiba roça-dar-no

'A que é dada por roça à Itajiba'

43. i-kó-mee'-byr-a

3-roça-dar-objeto-nom

'A quem é dada a roça'

(LEMOS BARBOSA, p. 383)

Uma outra evidência para a nossa proposta de análise vem do fato de que o nome incorporado pode coocorrer com o prefixo possessivo genérico de $3^{\mathrm{a}}$ pessoa, como em (44). $T$ - é possessivo de classe e indica que o possuidor é genérico de gente e não de animal. $T$ - não indica um indivíduo específico. Sendo assim, (44) não pode significar: "eu dei os filhos do meu marido" em que "marido" tem interpretação de possuidor:

Tupinambá

44. a-t-‘ay-meeng xe-mena 1sg-3gen-filho-dar 1sg.poss-marido

' Eu dei filhos a meu marido'

(LEMOS BARBOSA, p. 206)

Podemos concluir que as construções com ascensão de possuidor envolvem o núcleo Aplicativo do tipo Baixo (assimétrica) que não é realizado fonologicamente. Sendo aplicativas assimétricas, somente o objeto aplicativo se comporta como objeto sintático, já que engatilha concordância verbal, pode ser nominalizado e ainda é passível de incorporação como qualquer outro objeto, conforme ilustram os dados a seguir:

Tupinambá

45a. pe-îo-ok nhandu-r-aba

2pl-3-arrancar ema-rel-pena

'Arrancai as penas da ema' 
b. pe-s-ab-ok nhandu

2pl-3-pena-arrancar ema

'Arrancai as penas à ema'

c. pe-nhandu-r-ab-ok

2pl-3-ema-rel-pena-arrancar

‘Arrancai as penas à ema’ (LEMOS BARBOSA, p. 207)

\section{Conclusão}

Neste trabalho, descrevemos e discutimos a natureza das construções aplicativas verificadas em Paumari, Guarani e Tupinambá, à luz da tipologia de Pylkkänen $(2000,2002)$ para os núcleos aplicativos.

Observamos que existem afixos específicos que se agregam apenas a verbos intransitivos, inclusive os inergativos. Propomos, então, que tais afixos são a realização fonológica dos núcleos Aplicativos Altos que, como denotam uma relação entre um indivíduo e um evento, podem licenciar objetos com papéis temáticos variados, como: comitativo, fonte, locativo, instrumento.

Mostramos ainda a existência de afixos que só se agregam a verbos transitivos e que denotam uma relação de transferência de posse direcional (de fonte) para (alvo/benefecativo) entre o objeto direto e o objeto aplicativo. Seguindo Pylkkänen (2002), sugerimos que estes são a manifestação do núcleo Aplicativo Baixo que libera objetos com papéis temáticos específicos, como fonte ou alvo/benefactivo. Verificamos também que as construções com ascensão do possuidor nas línguas Tupi-Guarani podem ser analisadas como aplicativas do tipo baixo cujo núcleo não é realizado fonologicamente. 


\section{Notas}

${ }^{1}$ Possessor Stranding é o termo adotado por Baker (1988) para descrever as construções em que o elemento possuído é incorporado ao verbo, deixando para trás o possuidor na posição de objeto direto, como o exemplo do Mohawk pode ilustrar: Mohawk

(i) wa-hi-nuhs-ahni:nu: John aor-1/3-casa-comprar John 'Eu comprei a casa de João'

${ }^{2}$ Lista de abreviações utilizadas no texto: abs.=absolutivo; acus.=acusativo; aor= aoristo; apl.=aplicativo; asp.=aspecto; aux.=auxiliar; caus. =causativo; class.=classificador; dem.=demonstrativo; erg.=ergativo; f.=feminino; foc=foco; gen=genérico; imp=imperativo;int.=intransitivo; inter.=interrogativo;it=iterativo; $\mathrm{m}=$ masculino; $\mathrm{md}=$ modo; obl=oblíquo; pass.=passado;poss=possessivo; pres.=presente; $\mathrm{ps}=$ passiva; refl=reflexivo; rel.=relacional; $\mathrm{sj}=$ =sujeito; sg.=singular; tran.=transitivo.

${ }^{3}$ Vezinho ( $v$ ) é o morfema que categoriza (=verbaliza) a raiz lexical, transformandoa em um verbo.

${ }^{4}$ Para Harley (2006), Causa introduz o argumento externo e pode também funcionar como categorizador das raízes lexicais.

${ }^{5}$ Segundo Baker (1988), o termo “ alçamento do possuidor” (Possessor Raising) é conferido a uma construção em que o possuidor se torna um objeto do verbo, assim como o nominal possuído. Neste caso, tem-se uma oração com dois objetos. Já o termo "ascensão do possuidor" (Possessor Stranding) é empregado quando o nominal possuído é incorporado ao verbo, deixando o possuidor para trás, na condição de objeto sintático.

${ }^{6}$ Nesta análise, o argumento externo é gerado como especificador de vP (vezinhoP).

${ }^{7}$ Uma interpretação para (12) poderia ser: "quebrou o óculos de Siegel que estava na posse de Rina (from the possession of Rina)."

8 “É perfeitamente possível analisar 'convidado' como um objeto afetado adicionado por um tipo de processo aplicativo, em vez de um possuidor de um nome incorporado... ‘convidado' não é meramente um possuidor em [15b], mas sim um objeto aplicativo [com papel semântico de] malefactivo...”

${ }^{9}$ Todos os dados do Paumari aqui apresentados foram extraídos de Chapman (1978) e Chapman e Derbyshire (1991).

${ }^{10}$ Em Chapman (1978) e Chapman e Derbyshire (1991) não se encontra referência ao 
termo aplicativo. As estruturas que tratamos aqui como aplicativas são denominadas comitativas ou benefactivas pelos autores, dependendo da interpretação semântica dos objetos envolvidos.

${ }^{11}$ Em Paumari, coexistem dois tipos de sistemas de marcação de caso: o ergativoabsolutivo e o nominativo-acusativo. No sistema ergativo, o sujeito transitivo recebe o sufixo- $a$ e o objeto, assim como o sujeito intransitivo, vem precedido por um demonstrativo. As ordens observadas são: SVO e VS. O verbo transitivo concorda com o sujeito em termos de pessoa e número. O sujeito intransitivo e o objeto engatilham concordância de gênero, expressa no sufixo de modo:

(i) Jamahi-a bi-anani-hi ida isai SVO

cachorro-erg 3-morder-md/f dem/f criança

'O cachorro mordeu a criança'

(ii) abini-hi ida arakava VS morrer-md/f dem /f galinha

'A galinha morreu'

As formas femininas do sufixo de modo e do demonstrativo são respectivamente: -hi e ida. As formas masculinas são -ha e ada, conforme indicam os exemplos a seguir:

(iii) asara-hi ida isai chorar-md/f dem/f criança

'A menina chorou'

(iv) asara-há ada isai choara- $\mathrm{md} / \mathrm{m}$ dem $/ \mathrm{m}$ criança

'O menino chorou'

No sistema nominativo, o objeto é marcado pelo sufixo-ra, enquanto o sujeito transitivo ocorre sem marcas de caso. A ordem verificada é SOV. Nenhum dos dois argumentos engatilha concordância verbal. A forma feminina é sempre empregada no sufixo verbal, independentemente dos traços de gênero do objeto:

(iii) mafo ho-ra anana-há'i-hi SOV

formiga 1sg-acus morder-asp-md

'A formiga me mordeu'

${ }^{12}$ O sufixo - $a$ do Paumari, além de expressar o caso ergativo, também é usado para indicar o caso oblíquo.

${ }^{13}$ Os dados do Guarani foram coletados pela autora entre os índios guarani (Mbyá) residentes na aldeia Boa Vista em Ubatuba, São Paulo. Os exemplos do Tupinambá foram extraídos de Lemos Barbosa (1956). 
${ }^{14}$ Em Lemos Barbosa (1956, p. 199), a tradução dada para (26b) é: 'Fizestes a criança ver o céu contigo?'. De acordo com a nossa análise, o objeto comitativo não é a $2^{\mathrm{a}}$ pessoa, mas sim "a criança”. Daí a nossa tradução diferir daquela sugerida pelo autor.

${ }^{15}$ Quando -r o ocorre em ambiente nasal, realiza-se como -no, devido a um processo de assimilação.

${ }^{16}$ Os autores chamam de benefactivas as aplicativas deste tipo.

17 “O paciente semântico, que é o objeto direto na oração transitiva comum, tornase o objeto demovido na construção bitransitiva benefactiva. Este pode ser marcado pelo enclítico -a do objeto demovido ou pode ocorrer em posição pós-verbal com um demonstrativo , mas sem nenhuma outra marca."

${ }^{18}$ Lemos Barbosa (1956) usa as duas posposições -“a” e “ de” - na tradução dessas construções em Tupinambá, provalvemente pela sua interpretação de transferência de posse.

${ }^{19}$ Note-se que Lemos Barbosa usa a preposição "a” e não “de” (posse) na tradução de (40) e (41).

\section{Referências Bibliográficas}

BAKER, M. C. Incorporation: A Theory of Grammatical Function Changing. Chicago: The University of Chicago Press, 1988.

BAKER, C. The Polysynthesis Parameter. Oxford: Oxford University Press, 1998.

CHAPMAN, S. Paumari derivational affixes. Brasília: SIL, 1978. Ms.

CHAPMAN, S.; DERBYSHIRE, D. C. Paumarí. In: DERBYSHIRE, Desmond; PULLUM, Geoffrey K. (Ed.). Handbook of Amazonian Languages. Berlim: Mouton de Gruyter, 1991.

DOURADO, L. Construções aplicativas em Panará. DELTA. São Paulo, v. 8, n. 2, 2002.

HARLEY, H. On the causative construction. In: MIYAGAWA, S.; S. MAMURO (Ed.). The Oxford Handbook of Japanese Linguistics. Oxford: OUP, 2008.

JEONG, Y. The landscape of applicatives. 2006. Tese (Doutorado) - University of Maryland, 2006.

LANDAU, I. Possessor raising and the structure of VP. Língua v. 107, 1999.

LEMOS BARBOSA, Pe. Curso de Tupi Antigo. Rio de Janeiro: Livraria São José, 1956. 
MARANTZ, A. No scape from syntax: don't try morphological analysis in the privacy of your own lexicon. In: DIMITRIADIS, A.; SIEGEL, L. et al. (Ed.). University Working Papers in Linguistics, v. 4, n. 2, 1997.

MCHOMBO, S. A. Chichewa (Bantu). In: SPENCER, A.; ZWICKY, A. M. (Ed.). The handbook of Morphology. Oxford: Blackwell, 1998.

PYLLKäNEN, L. What applicative heads apply to?. Working Papers in Linguistics. Universidade da Pensilvânia, v. 64, 2000.

PYLKKANEN, L. Introducing arguments. 2002. Tese (Doutorado) - MIT, 2002.

VIEIRA, M. M. D. A natureza das sentences possessivas em Mbyá-Guarani. In: QUEIXALÓS, F. (Org.). Des noms et de verbs en Tupi-Guarani: état de la question. LINCOM Studies in Native American Linguistics, 37. Muenchen, LINCOM EUROPA, 2001. 